\title{
Design of Negative Resistance Oscillator with Rocord Low Phase Noise
}

\author{
Ayoub Malki ${ }^{*}$, Jamal Zbitou ${ }^{2}$, Larbi El Abdellaoui ${ }^{3}$, Mohamed Latrach ${ }^{4}$, \\ Abdelali Tajmouati ${ }^{5}$, Ahmed Errkik ${ }^{6}$ \\ 1,2,3,5,6 LMEET Laboratory FST of Settat Hassan 1st University, University Complex \\ Casablanca Road, Km 3.5, B.P: 577 Settat, Morocco \\ ${ }^{4}$ Microwave group ESEO Angers France, 10 BdJeanneteau-CS 9071749107 ANGERS CEDEX 2 \\ ${ }^{*}$ Corresponding author, e-mail: ayoub.malki88@gmail.com ${ }^{1}$, zbitou3676@gmail.com ${ }^{2}$
}

\begin{abstract}
The aim of this paper is to use a new design of a negative resistance microwave oscillator in order to fabricate oscillator with very good performance in terms of output power, efficiency, stability and phase noise. In this study the new concept of oscillator using distributed resonator and micro-strip circuit elements improve performances of our structure. A micro-strip microwave oscillator with low phase noise based on an NPN silicon planar epitaxial transistor has been designed, fabricated, and characterized. In this design, each step has been conducted by using Advanced Design System (ADS) and following a theoretical study which enable to optimize the different performances of the whole circuit. The oscillator produce a sinusoidal signal with spectrum power of $12.25 \mathrm{dBm}$ at $2.45 \mathrm{GHz}$ into $50 \Omega$ load when polarized at $V c c=15 \mathrm{~V}$ with $D C$ to RF efficiency of 16 . The obtained phase noise of $-120 \mathrm{dBc} / \mathrm{Hz}$ at $100 \mathrm{~Hz}$ offset is the result of the use of high $Q$ factor resonator and the depth study of the parameters of the oscillator. Simulation and measurement results are in good agreement.
\end{abstract}

Keywords: Oscillator, Negative resistance oscillator, Phase noise, Resonator

Copyright () 2018 Universitas Ahmad Dahlan. All rights reserved.

\section{Introduction}

The transceiver systems are becoming more complex to preserve the quality of information to transmit. These systems require local oscillator with very precise performance, the design of these oscillators is an attractive research field. The progress made in the microwave field have led to gradually replace the old type of oscillators with a more appropriate technology in terms of size and consumption. Today they take place in most communication equipment. Optimization of their characteristics is a critical point in the context of achieving the emission systems.

Oscillators are structures that can be found in any electronic system since (GSM, radio communication, spacial communication, radar, electronic war and instrumentation) it is up to them to create the electrical signal in different frequency ranges. Then the quality of any system depends directly on the performance of the oscillator.In the other hand the technological tendency, the requirements of the regulations and the speed of development of the telecommunications have increasingly forced to fabricate oscillator with very precise performances in term of size, consumption, phase noise, stability and output power.

To obtain very heighperformance several methods are used to achieve this goal, among theses methods we distinguish for instance: the methodbased on the negative resistance and the methodbased on the looped system. The configuration of looped system technique can only be used for a resonator with a single type of resonance. Also we have no control over the established state of the circuit, especially of other eventual instability that sought at the desired frequency. The negative resistance concept is more understandable for reflected oscillator, although it can be applied for all types of oscillator. The harmonic balance simulation permits to determine accurately the established state of the circuit. That is why we are interested in this project to the reflected oscillator in the form of negative resistance system.

Therfore in the present paper a new design of negative resistance oscillator are used for phase noise reduction, more stability of frequency, height efficiency of our oscillator and significated output power. Than the caracteristique of our oscillator show that we have 
developed very performed structure using NPN silicon planar epitaxial transistor as active device. Theses results has been reached because each step has been analyzed deeply to optimize the different performance of the whole circuit especially the phase noise. More details of the proposed oscillator design procedure, simulated and measured results are presented and discussed [1-3].

\section{Design Procedure}

A microwave oscillator has three separates blocks: Resonator for choosing the frequency of oscillation, an amplifier (constituted of active device, feedback element and bias network) which operates at the desired frequency and an output load connected to the oscillator via a matching impedance module. The block diagram of the microwave oscillator with its different parts is shown in Figure 1 [4-6].

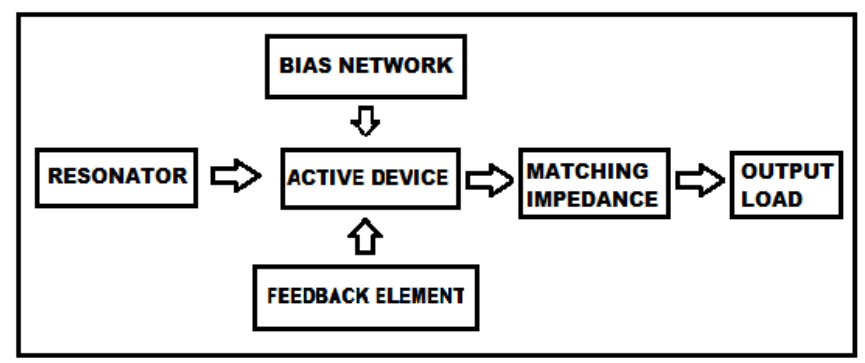

Figure 1. Basic oscillator model

The first way to understand the operation of an oscillator is to see the circuit as an amplifier and the passive circuit as a filter as shown in Figure 2.

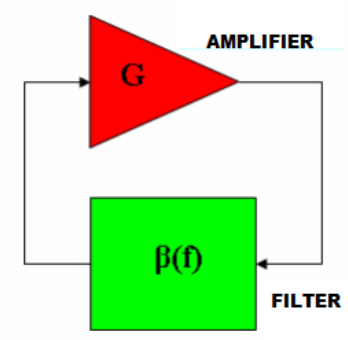

Figure 2. Oscillator as feedback loop

In this case, the oscillator may be understood as a selective noise amplifier whose operation can be described as follows: The intrinsic noise of the electronic circuits is amplified by the active part of the oscillator. The signal resulting from the amplification of the noise would be brought to the input of the amplifier phase-shifted by $180^{\circ}$ in order to avoid any unstability of the system. For an oscillator, this unstability is necessary. The feedback loop selects the frequency at which oscillations are desired and re-injects the output without phase shift [7-8]. So the noise is amplified until a signal oscillating at the frequency determined by the filter. Barkhausen described this step by two conditions on the gain and phase of the loop:

$$
\begin{aligned}
& \left\|\beta\left(f_{0}\right)^{\star} G\right\|=1 \\
& \Phi\left(\beta\left(f_{0}\right)^{\star} G\right)=0
\end{aligned}
$$


To have a signal with the requirements witch we look for, we have to satisfy the two barkausen conditions. For that we can modulate the oscillator in the form of the negative resistance to benefit from its advantages. The concept of negative resistance as shown in Figure 3 consists to see the oscillator as negative resistance compound of active device $\left(R_{a} ; X_{a}\right)$ and load $R_{L} X_{L}$ ( $X_{L}$ to choose frequency of oscillation and $R_{L}$ :loss resistance).

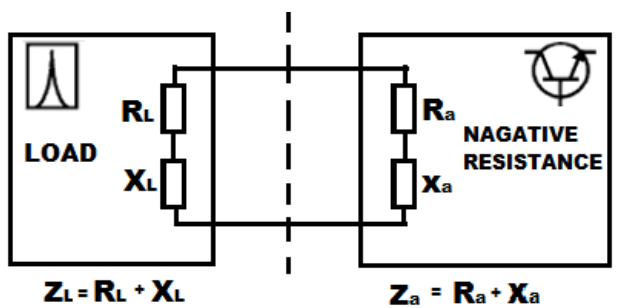

Figure 3. Block diagram of negative resistance oscillator

The circuit is intrinsically oscillating but its oscillations disappear after a certain period of time due to the dissipation of the circuit energy in the resistor. The active device must supply an amount of energy equal to the energy dissipated in the parasitic resistance and the resonator must compensate the imaginary impedance of the active device at the desired oscillation frequency.Barkhausen condition's become (3) and (4).

$$
\begin{aligned}
& R_{I N}+R_{L}=0 \\
& X_{I N}+X_{L}=0
\end{aligned}
$$

During the remaining procedure items we will have two objectives: to have a very high negative resistance of the active device and a minimal dissipation of energy at the level of the resonator at a given frequency $2.45 \mathrm{GHz}$. To produce a maximum negative resistance via the active device (transistor) we must to make a transistor in unstable area. In this example we have chosen an acceptable transistor's gain and power related to the operating frequency and the topology that will give appropriate performances.

Based on the theory above, taking into account the scattering parameter of the transistor at $2.45 \mathrm{GHz}$ with common base configuration and the value of $\mathrm{k}$ factor $(|\mathrm{K}|>1)$, we have chosen a transistor which can function at a stable area.

$$
K=\frac{1-\left|S_{11}\right|^{2}-\left|S_{22}\right|^{2}+\left|S_{11} S_{22}-S_{12} S_{21}\right|^{2}}{2\left|S_{21} S_{12}\right|}
$$

\section{$\mathrm{K}$ : The stability factor of the transistor}

$[\mathrm{s}]=\left[\begin{array}{ll}S_{11} & S_{11} \\ S_{11} & S_{11}\end{array}\right]$ : The s-parameter of the transistor.

Therefore we can try a different configuration, and we can add a feedback structure to have the stability factor less than the unit $(|\mathrm{K}|<1)$ and the module of return loss greater than the unit in the device input port $(|S 11|>1)$. For this study we have chosen the common emitter configuration with a micro-strip feedback structure to modify the device scattering parameters. By using ADS, we can simulate the active device with stabfact to determine the stability factor $\mathrm{k}$. The resulting $k$ factor versus frequency is shown in Table 1 [9-10].

Table 1. k Factor for the Active Device

\begin{tabular}{cc}
\hline FREQUENCY & STABFACT: $\mathrm{k}$ \\
\hline $2.40 \mathrm{GHz}$ & -0.753 \\
$2.45 \mathrm{GHz}$ & -0.836 \\
$2.50 \mathrm{GHz}$ & -0.786 \\
\hline
\end{tabular}


Transistor can't work without bias. The study of the bias transistor enable to fix its operating point and improve its paramaters. Consequently we can know its $S$ parameter, especially when the manufacturer gives only s-parameter to specific bias points.

In this project we have chosen a bias form's with lower consumption of power. using the ADS software program, we have performed an optimization study which gave us the following bias resistors: R1=150 KOhm, R2=15000 Ohm, R3=1500 Ohm. By optimization and theoretical study we have obtained the lowest possible real part of the output impedance of the transistor $\mathrm{R}_{\mathrm{a}=}=632.295$ shown in Figure 4 .

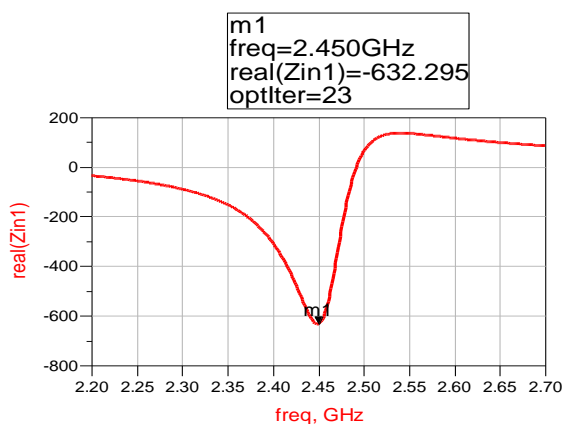

Figure 4. Result of the simulation of parameter real (Z1) under ADS

Once all the previous steps are completed, namely the active element and the biasing circuit to produce maximum negative resistance at $2.45 \mathrm{GHz}$. The next step is to choose a structure (resonator) that provides the desired operation frequency.

The resonator is the essential component of the oscillator. Its selectivity allows to fix the oscillation frequency, and its quality factor $Q$ dominates the performance of phase noise. The resonator losses are not null but are canceled with the negative resistance created by the active device. From the various existing topologies, a micro-strip line can be chosen for its size and its higher unloaded $Q$ [15].

The last step is to design matching impedance. The problem with the matching impedance is its resistance $R_{\text {IMP. }}$. To design $R_{I M P}$ the best way is to select its value for maximum oscillator power. For frequencies around the frequency of oscillation we can see that $R_{\text {IMP }}$ is only a function of the amplitude $A$ of $i(t)$. Kurokawa gives an appropriate value of $R_{I M P}$, which maximizes the oscillator power is:

$$
R_{I M P}=-R_{a} / 3
$$

In order to realize the output match impedance with the above condition, we perform a structure under ads and by optimization we have an adaptation cell with an input impedance which satisfies Equation 6. After we have added a filter at the output of the oscillator to eliminate harmonics we have obtained the final circuit as shown in Figure 5.

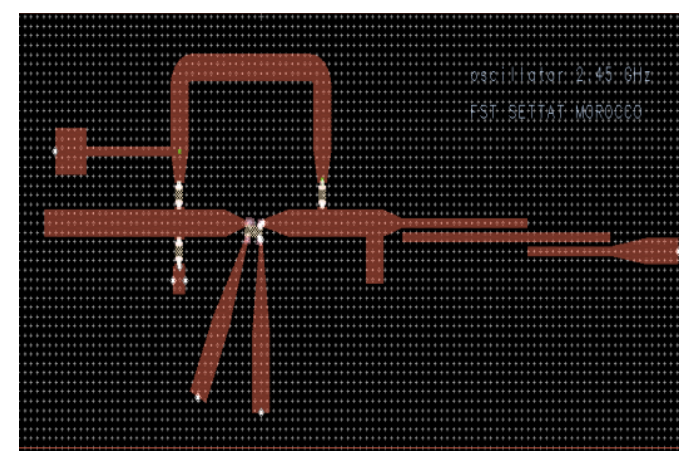

Figure 5. The layout of negative resistance microwave oscillator obtained through ADS 


\section{Results and Analysis}

We want to know the performance of our circuit for phase noise, quality of the signal and output power, for that Figure 6. Figure 7 and Figure 8 show successively the typical output spectrum of the oscillator, steady-state, and the phase noise of the output signal. The oscillation frequency is $2.45 \mathrm{GHz}$ in ISM band. The oscillator reaches a significantly low phase noise of $120 \mathrm{dBc} / \mathrm{Hz}$ at $100 \mathrm{~Hz}$ offset. Also the oscillator delivers an output power of $12.25 \mathrm{dBm}$ with high DC to RF conversion efficiency of $16 \%$.

According to the Figure 9, the addition of the bandpass filter has improved the distortion rate of the output signal by the attenuation of undesirable harmonics. The first harmonic equals $18.056 \mathrm{~dB}$ and the second harmonic equals $68.605 \mathrm{~dB}$. So the distortion rate $\mathrm{d}=2.9 \%$.

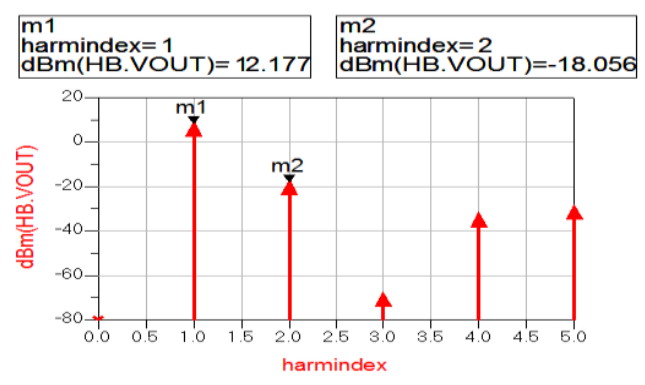

Figure 6. Oscillator output power spectrum

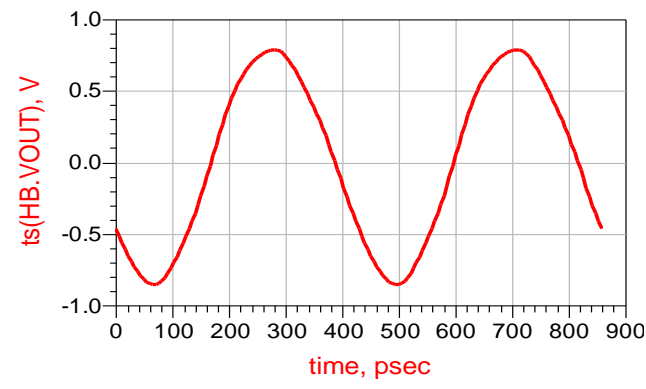

Figure 7. Output signal in time domain of the oscillator at $2.45 \mathrm{GHz}$

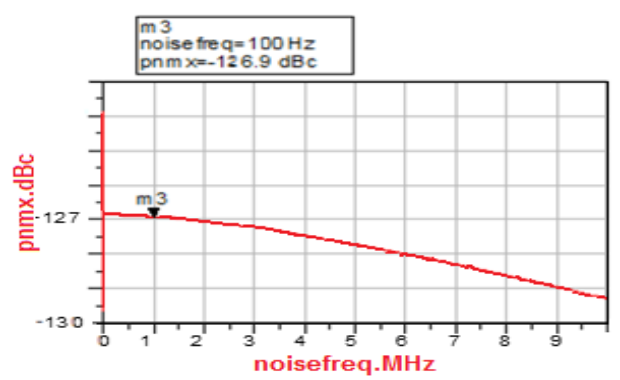

Figure 8. Phase noise prediction of the microwave oscillator design

The oscillator was fabricated on the Teflon substrate with the dielectric constant of 10 , the metal thickness of $0.018 \mathrm{~mm}$, and height is $0.64 \mathrm{~mm}$; as hybrid microwave integrated circuit structure. All the circuit components are in SMD (Surface Mount Device) package. Figure 9 illustrates the photograph of the completed fabricated oscillator. The size of the ground is less than $0.5 \mathrm{~mm}$ on a side which equates to less than $1 / 10$ of wavelength at $2.45 \mathrm{GHz}$ and determined to be reasonable ground.

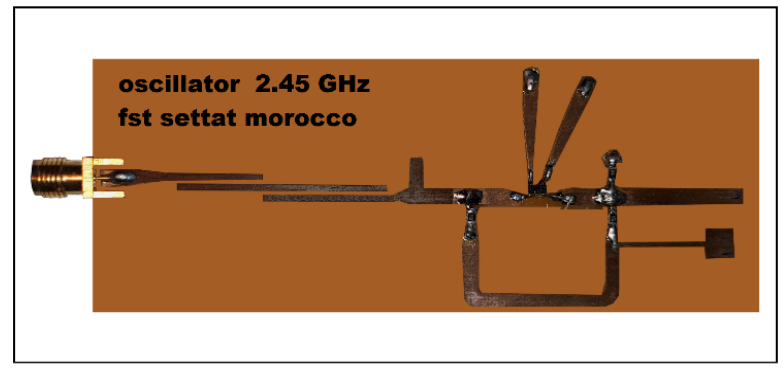

Figure 9. Photograph of the fabricated microwave oscillator 
The phase noise of the circuit was measured using Agilent 5505A phase noise measurement system by the method of the two channel cross-correlation technique. The purpose of the phase noise measurement is to quantify the short term stability of an oscillator. Phase noise measurements are perhaps the most sensitive measurements being done at microwave frequencies. From Figure 10 the phase noise levels of negative resistance oscillator with the microstrip resonator is $-120 \mathrm{dBc} / \mathrm{Hz}$ at $100 \mathrm{~Hz}$ at an oscillation frequency of $2.45 \mathrm{GHz}$.

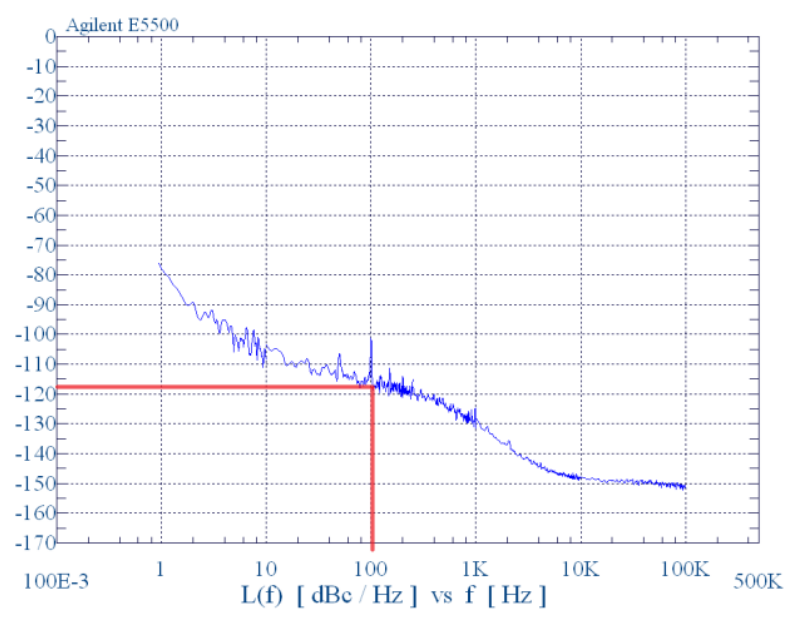

Figure 10. Measured phase noise of microwave oscillator

The microwave oscillator is characterized in frequency domain using Agilent E4440A PSA series spectrum analyzer. The oscillator delivers $12.25 \mathrm{dbm}$ at $2.45 \mathrm{GHz}$ into $50 \Omega$ load when polarized at $V=15 \mathrm{~V}$, with dc to RF efficiency equal $16 \%$. Figure 11 shows the oscillator's power spectrum measured. The second harmonic was founded $30 \mathrm{~dB}$ below the fundamental signal.

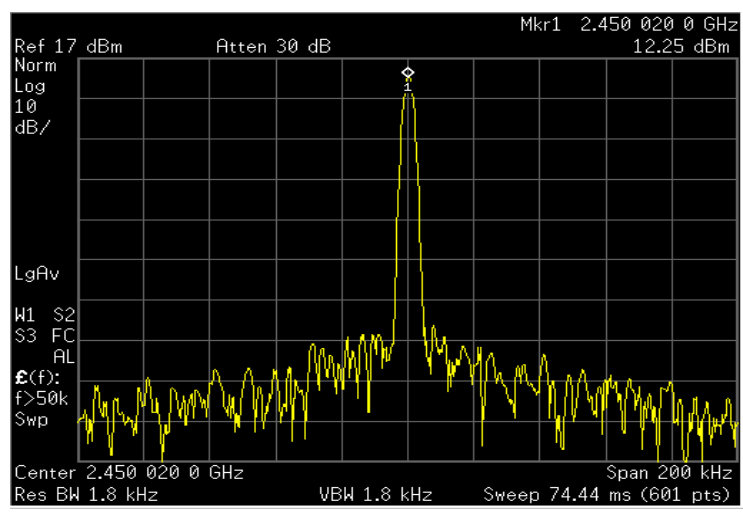

Figure 11. Measurement result of microwave oscillator

Tektronix DPO 7245 digital phosphor oscilloscope with bandwidth $2.5 \mathrm{GHz}$ is used for the measurement of the output signal of the circuit in time domain. The oscillator produces an output signal in time domain at $2.45 \mathrm{GHz}$. The output voltage has ideal sinusoidal shape with peak to peak voltage swing of $2.14 \mathrm{~V}$.

The simulated and measured parameters of our oscillator present approximately the same results in term of frequency and output power and some offset in phase noise $(6 \mathrm{dBc})$. 
This offset is normal due to the fabrication, approximations taken by ADS especialy phase noise and the quality of components.

Through published results of some scientific reports on microwave oscillator designs [11-17] we observe that the best reached results are about: phase noise $-101 \mathrm{dBc} / \mathrm{hz}$ efficiency 15. If we compared these results with ours (phase noise $-120 \mathrm{dBc} / \mathrm{hz}$ efficiency 16 ) we can conclude that this oscillator has record results in term of phase noise, better results in term of efficiency, good output power and frequency accuracy. These results were the product of thorough study of each block of the oscillator and especially the interaction between these blocks.

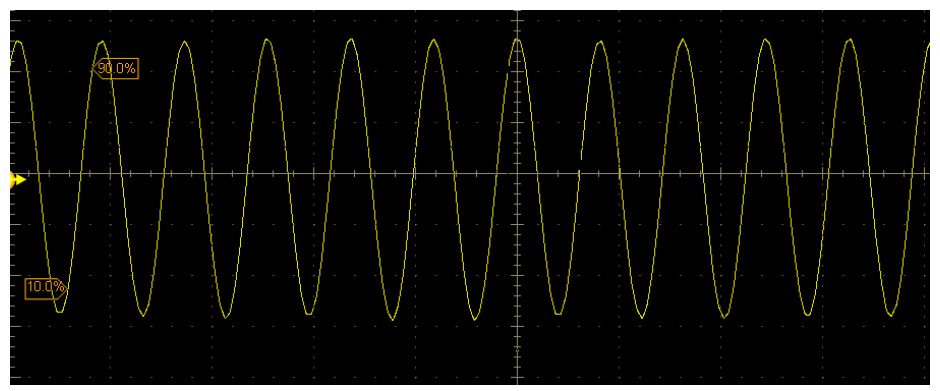

Figure 12. Measured output signal in time domain of the oscillator

\section{Conclusion}

As a conclusion, we can deduce that we have developed a novel structure negative resistance oscillator at $2.45 \mathrm{GHz}$ in ISM band with a record low phase noise performance, high dc to RF efficiency, output power, distortion and frequency accuracy. This circuit has been designed and adjusted by using a theoretical step and optimization tools applied on matching impedance, resonator, active device and Bias networks. The fabricated oscillator has a phase noise of the output signal around $-120 \mathrm{dBc} / \mathrm{Hz}$ at $100 \mathrm{~Hz}$, an output power at $2.45 \mathrm{GHz}$ equal to $12.72 \mathrm{dBm}$, distortion rate equal to and DC to RF efficiency equal to $16 \%$. The different steps followed to validate this oscillator can be used to tune the frequency and to achieve an oscillator in other frequency bands.

\section{Acknowledgment}

We thank Mr. Mohamed LATRACH Professor in ESEO, Engineering Institute in Angers, France for allowing us to use all the equipments and software available in his laboratory

\section{References}

[1] MM Elsherbini, MF Elkordy, AM Gomaa. Design and Simulation for UHF Oscillator using SAWR with Different Schematics. Indonesian Journal of Electrical Engineering and Computer Science (IJEECS). 2016; 1(2): 294-299.

[2] Kengo Kawasaki, Takayuki Tanaka, Masayoshi Aikawa. Ku band Second Harmonic N-Coupled Push-Push Oscillator Array using Microstrip Resonator. Proceedings of the IEEE MTT-S on Microwave Symposium Digest. Anaheim. 2010:1182-1185

[3] T Ohira. Rigorous Q-factor formulation for one- and two-port passive linear networks from an oscillator noise spectrum viewpoint. IEEE Transactions on Circuits and Systems II: Express Briefs. 2005; 52(12): 846-850.

[4] DM Pozar. Editors. Wiley. Microwave engineering. 3 Edition. India.1989.

[5] Bhan u Shrestha, N Kim. Low phase noise microwave oscillator using meander spurline resonator for X band application. Indian journal of engineering materials sciences. 2011; 18: 381-384.

[6] Bhavanabenakaprasad, Salah sharabi, K. Elgaid. RF and Microwave oscillato r design using p-HEMT transistor. International journal of scientific and research publication. 2014; 4(8): 1-7.

[7] A Hajimiri, TH Lee. Editors. Norwell. The design of low noise oscillator. 1999.

[8] K Chang, I Bahl, V Nair. Editors. Wiley. RF and microwave circuit and component design for wireless systems. Texas. 2002. 
[9] Achmad Munir, Endon Bharata. Self oscillating mixer with dielectric resonator for low noise block application. TELKOMNIKA (Indonesian Journal of Electrical and Engineering). 2011; 9(2): 351-356.

[10] Khairun Nisa' Minhad, Zainab Kazemi, Mamun Bin IbneReaz, Jubayer Jalil, Noorfazila Kamal. Design of a Current Starved Ring Oscillator Based VCO for Phase-Locked Loop. TELKOMNIKA (Indonesian Journal of Electrical and Engineering). 2014; 12(9): 6667-6672.

[11] RA Pucel, R Bera, D Masse. Experiments on integrated gallium -arsenide FET oscillators at $X$ band. Electronics letters.1975; 11(10): 219-220.

[12] O Ishihara, T Sawano, M Nakatani. Highly stabilized GaAs FET oscillator using a dielectric resonator feedback circuit in 9-14 GHz. IEEE transaction on microwave theory and techniques. 1980; 28(8): 817-824.

[13] S Chen, S Tadayon, T Ho, K Pande, P Ria, J Adair, M Ghahrenani. U-band MMIC HBT DRO.IEEE microwave and guided wave letters.1994; 4(2): 50-52.

[14] H Jacobsson, S Gevorgian, M Mokhtari, C Hedenas, B Hansson, T Lewin, H Berg, W Rabe, A Schuppen. Low phase noise low power IC VCO for $5-8 \mathrm{GHz}$ wirless application. IEEE MTT-S integration microwave symposium. Boston. 2000; 48(12): 723-726.

[15] Hun-Wah Fan, KKM Cheng. New method in characterizing the nonlinear current model of MESFET using single-tone excitation. IEEE MTT-S International on Microwave Symposium Digest. Boston. 2000: 449-452.

[16] D Kuylenstiena, S lai, M Bao, H Zirath. Design of low phase noise oscillators and wideband Ward VCOs in InGap HBT technology. IEEE Transactions on Microwave Theory and Techniques. 2012; 60 (11): 3420-3430.

[17] Madureira, N Deltimple, E Kerherve. Design and measurement of class EF2 power oscillator. Electronics Letters. 2015; 51(10): 5-14. 